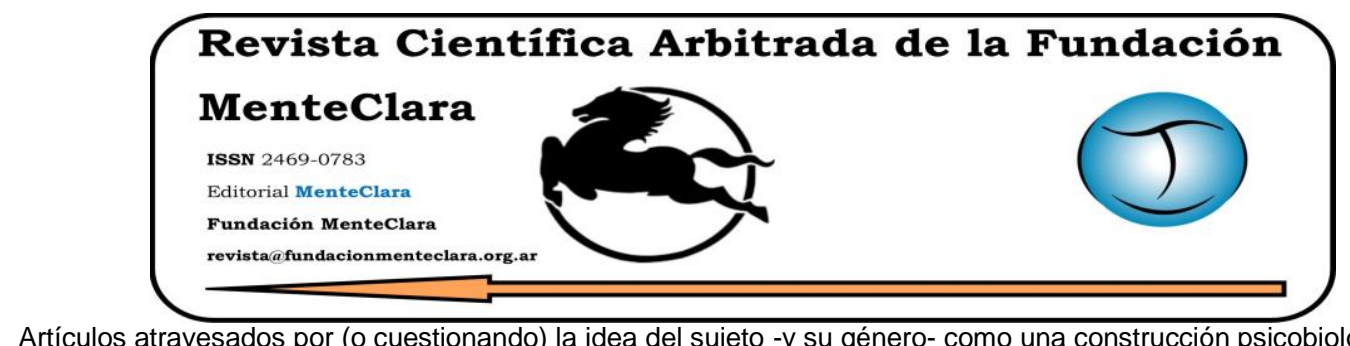

Artículos atravesados por (o cuestionando) la idea del sujeto -y su género- como una construcción psicobiológica de la cultura. Articles driven by (or questioning) the idea of the subject -and their gender- as a cultural psychobiological construction

Vol. 2 (2), 2017

ISSN 2469-0783

https://datahub.io/dataset/2017-2-2-e31

\title{
LA SALUD MENTAL EN LA PSICOLOGÍA DE LA RELIGIÓN Y DE LA ESPIRITUALIDAD
}

\section{MENTAL HEALTH WITHIN THE PSYCHOLOGY OF RELIGION AND SPIRITUALITY}

Hugo Simkin hugosimkin@psi.uba.ar

Universidad de Buenos Aires (UBA), Argentina.

Cómo citar este artículo / Citation: Simkin H. (2017). "La salud mental en la psicología de la religión y de la espiritualidad". Revista Científica Arbitrada de la Fundación MenteClara, 2(2), 169-204. DOI: 10.32351/rca.v2.2.33

Copyright: (C) 2017 RCAFMC. Este artículo de acceso abierto es distribuido bajo los términos de la licencia Creative Commons Attribution-Non Commercial (by-cn) Spain 3.0. Recibido: 30/08/2017. Aceptado: 30/09/2017 Publicación online: $30 / 10 / 2017$

Conflicto de intereses: Ninguno que declarar.

Este trabajo fue financiado en el marco de los proyectos de investigación UBACyT 20020150200259BA y PICT-2016-4147 acreditados por la Universidad de Buenos Aires (UBA) y la Agencia Nacional de Promoción Científica y Tecnológica (ANPCyT).

\section{Resumen.}

Si bien la psicología se ha interesado por los fenómenos religiosos y espirituales desde sus origines, recién en la década del ochenta se constituye la psicología de la religión y de la espiritualidad como un área específica de la psicología. En este marco, entre las principales líneas de investigación, numerosos autores se han interesado en explorar el modo en que la religiosidad y la espiritualidad se asocian a la salud mental. Sin embargo, a pesar de la considerable cantidad de estudios que abordan el tema, en la literatura especializada aún se evidencia una amplia diversidad de resultados contradictorios. El presente trabajo se propone revisar los antecedentes vinculados al modo en que se han asociado estas variables, atendiendo a los debates teórico-epistemológicos que podrían contribuir a explicar las enormes diferencias que se observan entre los distintos hallazgos empíricos. 


\begin{abstract}
.
Although psychology has been interested in religious and spiritual phenomena since its origins, it was not until the 1980s that the psychology of religion and spirituality was established as a specific psychological field. Within this framework, among the main lines of research, numerous authors have been interested in exploring the way in which religiosity and spirituality are associated with mental health. However, despite the considerable number of studies that address the issue, a wide diversity of mixed results is still evident in the literature. The present work intends to review the antecedents linked to the way in which these variables have been associated, taking into account the theoretical-epistemological debates that could contribute to explain the enormous differences that are observed between the different empirical findings.
\end{abstract}

\title{
Palabras Claves/ Keywords
}

Religiosidad; espiritualidad; salud mental; Religiosity; spirituality; mental health 


\section{Introducción}

Si bien la psicología se ha interesado tempranamente en los fenómenos espirituales y religiosos, la psicología de la religión y de la espiritualidad se constituye como un área especifica dentro de la disciplina recién a mediados de la década del ochenta. En este marco, la relación entre la religiosidad, la espiritualidad y la salud mental representa una de las lineas de investigación que ha recibido mayor atención por parte de la comunidad académica. Sin embargo, a pesar de la considerable cantidad de artículos académicos que se han publicado sobre el tema, se observan importantes limitaciones en cuanto a la claridad conceptual con la que estos términos se definen en la literatura especializada. Estos obstáculos afectan directamente el desarrollo de técnicas de evaluación que permitan su relevamiento empírico. A su vez, la enorme disparidad en el modo en que se han operacionalizado los constructos numinosos impacta directamente en la posibilidad de sistematizar los resultados reportados en los distintos estudios.

El presente trabajo se propone relevar el estado del arte relativo al vínculo entre la espiritualidad, la religiosidad y la salud mental en la psicologia de la religión, atendiendo a las limitaciones mencionadas en cuanto al modo en que estos constructos suelen definirse y evaluarse. A tal efecto, en primer lugar se introduce al campo de la psicología de la religión y la espiritualidad. En segundo lugar, se revisan algunas de las definiciones de los términos espiritualidad y religiosidad propuestas en psicología de la religión. En tercer lugar se resumen las principales categorias con las que se define la salud mental desde una perspectiva psicológica. Posteriormente, se sintetizan algunos de los principales enfoques que intentan explicar el vínculo entre la religiosidad, la espiritualidad y la salud mental. En quinto lugar, se revisan los antecedentes que procuran examinar el vínculo entre la religiosidad, la 
espiritualidad y la salud mental, especialmente a la luz de los aportes de Koenig, King y Carson (2012). A modo de ejemplo, se abordaran específicamente los trabajos relativos a los trastornos del estado de ánimo, los trastornos de ansiedad y al bienestar subjetivo. Finalmente, se describen las principales limitaciones teóricas y metodológicas para la sistematización de los distintos estudios que exploran el tema.

\section{Psicología de la Religión y de la Espiritualidad: un campo en expansión.}

Si bien el fenómeno religioso-espiritual ha sido objeto de estudio de la psicologia desde sus inicios (Allport, 1950; James, 1902), a partir del trabajo de Gorsuch (1984), la cantidad de artículos publicados y el número de revistas especializadas en el tema se han incrementado considerablemente (Belzen, 2015; Belzen \& Hood, 2006; Muñoz, 2004). Entre las primeras revistas especialmente dedicadas a temáticas religiosas pueden mencionarse Review of Religious Research, editada por primera vez en el año 1959, y Journal for the Scientific Study of Religion, publicada originalmente en 1961. Actualmente existen numerosas revistas especializadas entre las que se destacan Journal of Psychology and Theology (1972), Journal of Psychology and Christianity (1981), International Journal for the Psychology of Religion (1991) y Mental Health, Religion \& Culture (1998). Esta creciente expansión de la cantidad de trabajos que indagan acerca de la religiosidad puede atribuirse al impacto que presenta este fenómeno para numerosos temas de estudio de gran relevancia para la psicología (Anderson, 2015; Argyle \& Beit-Hallahmi, 1975; Aten, O'Grady, \& Worthington, 2011; Kirkpatrick, 2013; Wesselmann \& DeSouza, 2015). Así, en el marco de dichas revistas se ha evaluado la relación entre la religiosidad, la espiritualidad y diferentes temas de investigación de esta disciplina, tales como los valores sociales 
(Fontaine, Duriez, Luyten, Corveleyn, \& Hutsebaut, 2005; Gorsuch, 1970), el prejuicio (Bernt, Kasanzew, \& López, 2007; Chalfant \& Peek, 1983; Spilka \& Reynolds, 1965), la autoestima (Benson \& Spilka, 1973; Krause \& Ellison, 2007; Smith, Weigert, \& Thomas, 1979), la personalidad (Kwilecki, 1986; Louden \& Francis, 1999; Melamed, Silverman, \& Lewis, 1974), el autoritarismo (Ji \& Ibrahim, 2007; Leak \& Randall, 1995), el proceso de socialización (Fichter, 1962; Jelen \& Chandler, 1996; Krauss et al., 2012), las actitudes (Cochran \& Beeghley, 1991; LaBouff, Rowatt, Johnson, \& Finkle, 2012) o la salud mental (Messay, Dixon, \& Rye, 2012; Watson et al., 2002). Además, desde el 2005 se han publicado numerosos handbooks específicos en el tema (De Souza, Francis, O’Higgins-Norman, \& Scott, 2009; Miller, 2012; Paloutzian \& Park, 2013; Pargament, 2013; Saroglou, 2014a). De acuerdo con Miller (2012), otro indicador del incremento de trabajos en el área es la creación de la división 36 de la American Psychological Association, cuya historia comienza a partir del interés que persigue la American Catholic Psychological Association por promover la profesionalización de la psicología en la comunidad católica, hasta su disolución en 1968. Según la autora, años más tarde, en 1971, esta organización adopta una postura de mayor ecumenismo y modifica su nombre a Psychologist Interested in Religious Issues (PIRI), consiguiendo en 1975 el estatuto de división dentro de APA bajo la denominación Psychology of Religion. Posteriormente, a partir del intenso debate en el campo acerca de la necesidad de distinguir el término espiritualidad de la noción de religiosidad, el nombre de la division se cambia a Society for the Psychology of Religion and Spirituality (Miller, 2012). De manera similar, la psicología de la religión y de la espiritualidad ha crecido en diferentes países fuera del contexto anglosajón (Hood \& Spilka, 2013; Nelson, 2012), tales como China (Chen, Wang, Weng, \& Wang, 2012; Dueck \& Han, 
2014; Lu \& Ke, 2012), Brasil (Esperandio \& Marques, 2014; Gomes Esperandio \& August, 2017; Paloutzian, 2017; Valle, 2017), o Turquía (Agilkaya \& Hood, 2012; Ağılkaya-Şahin, Streib, Ayten, \& Hood, 2015; Hökelekli, 2013).

\section{Espiritualidad y religiosidad: hacia una definición psicológica de}

\section{los términos.}

A lo largo de la historia de la disciplina se han identificado más de 30 definiciones diferentes para cada término (Hill et al., 2000; Koenig, 2008; Oman, 2013; Piedmont, 2013), al punto que las corrientes principales en psicologia han criticado que estas podrian reflejar las propias creencias de los investigadores antes que a constructos empíricamente observables (Musick, Traphagan, Koenig, \& Larson, 2000). Tal como se ha señalado, inicialmente ambas nociones fueron utilizadas indistintamente por los investigadores (Zinnbauer et al., 1997). En un segundo momento, fueron planteadas en términos opuestos: una religiosidad estática, institucional y objetiva, en oposición a una espiritualidad dinámica, personal, y subjetiva (Zinnbauer, Pargament, \& Scott, 1999). Posteriormente, se ha observado que la espiritualidad y la religiosidad no representan categorias opuestas, sino que resultan complementarias, de modo que las personas pueden considerarse a la vez religiosas y espirituales, tanto como espirituales pero no religiosas (Zinnbauer $\&$ Pargament, 2005). En la actualidad, una de las perspectivas de mayor relevancia es la propuesta por Piedmont (2012), quien sugiere definir la espiritualidad y la religiosidad en el marco del modelo y la teoría de los cinco factores de la personalidad (Costa \& McCrae, 1980; McCrae \& Costa, 1996, 2012). Desde esta perspectiva, la espiritualidad puede considerarse como una motivación innata que orienta y guía el comportamiento humano en el esfuerzo de construir un sentido más amplio de significado personal en 
un contexto escatológico (Piedmont, 2012), es decir, del destino del hombre luego de su muerte (Sayés, 2006). La religiosidad, por su parte, remite al modo en que la espiritualidad se desarrolla y se expresa a través de una organización comunitaria o social (Piedmont, 2004, 2012). De acuerdo con Piedmont (2012), la religiosidad no es considerada como un constructo motivacional o rasgo de la personalidad, sino que se define como un sentimiento, concepto que toma de autores clásicos de la psicología como Ruckmick (1920) y Woodworth (1940), para quienes éstos reflejan tendencias emocionales que se desarrollan a partir de las tradiciones sociales y de las experiencias educativas. Siguiendo a Piedmont (2012), si bien los sentimientos pueden ejercer una poderosa influencia sobre los pensamientos y los comportamientos, estos no representan cualidades innatas, como es el caso de la espiritualidad. Por eso, la expresión de sentimientos (e.g. prácticas religiosas) puede diferir de acuerdo a cada cultura (Piedmont, Kennedy, Sherman, Sherman, \& Williams, 2008) y ser más susceptible de cambiar o modificarse (Piedmont, 2004). Así, mientras que la espiritualidad se percibe como un atributo universal del individuo, como un rasgo de la personalidad (DyLiacco, Piedmont, Murray-Swank, Rodgerson, \& Sherman, 2009; MacDonald et al., 2015; Piedmont, 1999), la religiosidad remite a un conjunto específico de sistemas de creencias, prácticas y valores centrados alrededor de marcos institucionales explícitamente pautados e inmersos en determinadas tradiciones sociales (Miller $\&$ Thoresen, 1999), y se encuentra sujeta a la influencia de la cultura y de la educación (Wilkins, Piedmont, \& Magyar-Russell, 2012). Para Piedmont (1999), si bien resulta posible reconocer similitudes entre la espiritualidad y algunos de los cinco factores no existe en el FFM un perfil que pueda reflejar las características de una persona con elevada espiritualidad. En consecuencia, con el objeto de evaluar si la espiritualidad constituye un 
factor de la personalidad, distintos estudios han factorializado diferentes escalas que evalúan la espiritualidad junto con los cinco factores, concluyendo que muchas de ellas definen factores independientes de las dimensiones del FFM (MacDonald, 2000; Piedmont, 1999; Rican \& Janosova, 2010; Simkin, 2017). Estos resultados motivan un intenso debate respecto de si se trata de un sexto factor del modelo (Piedmont, 2012; Saroglou, 2011, 2014b).

\section{Salud mental desde una perspectiva psicológica.}

Desde sus inicios, el interés principal de la psicología clínica se focalizó en la evaluación y el tratamiento de los sintomas psicopatológicos (Maddux, 2008; Seligman \& Csikszentmihalyi, 2000), tal como se clasifican en el Manual Diagnóstico y Estadístico de los Trastornos Mentales de la Asociación Estadounidense de Psiquiatría, que actualmente se encuentra en su quinta edición (American Psychiatric Association, 2013). Sin embargo, si bien el interés por estudiar los aspectos positivos de la experiencia humana ha estado presente desde los inicios de la disciplina (James, 1890), con el correr de los años, a partir del surgimiento de la psicologia positiva ha crecido el interés por estudiar, no solo los déficits de las personas, sino también sus fortalezas (Diener, 2009; Myers, 1993; Seligman, 2002; Seligman \& Peterson, 2003). Desde este enfoque, la salud mental no sólo se define como la ausencia de síntomas psicopatológicos (e.g. depresión, ansiedad), sino también por la experiencia de bienestar (Diener, Oishi, \& Lucas, 2009; Lamers, 2012; Lamers, Westerhof, Glas, \& Bohlmeijer, 2015). Estos trabajos han contribuido a formular las nociones de bienestar psicológico y de bienestar subjetivo, entre otras (Diener, 2000; Efklides \& Moraitou, 2013). 
Las relaciones especificas entre la espiritualidad, la religiosidad y la salud mental han despertado el interés de numerosos investigadores (Brown, Chen, Gehlert, \& Piedmont, 2013; Ciarrocchi, 2012; Greenfield, Vaillant, \& Marks, 2009; Myers, 2008; Piedmont, 2009). Uno de los primeros autores en explorar la relación es Galton (1872), quien concluye que no existen diferencias en el estado de la salud entre las personas que rezan y las que no rezan. Posteriormente, Freud (1979) considera que la religiosidad contribuye a la domesticación de los instintos destructivos, aunque puede presentar un impacto negativo cuando implica el sometimiento de unas personas sobre otras. Para Ellis (1986) la religión resulta negativa para la salud mental dado que atenta contra la libertad del ser humano. De acuerdo con Jung (1938) la devoción religiosa puede ser positiva para la salud mental, al punto que considera la "Deidad" dentro de sus arquetipos. Allport (1950) introduce las categorias de religiosidad madura e inmadura para distinguir aquellas personas que presentan un interés genuino por la religión, de aquellas que la emplean para alcanzar ciertos objetivos personales, como poder o estatus social. En la actualidad, distintos autores han intentado comprender diferentes aspectos específicos de la espiritualidad y de la religiosidad que se asocian una mejor o peor salud mental (Dein, Cook, Powell, \& Eagger, 2010; Huguelet \& Koenig, 2009; Idler et al., 2009; Koenig, King, \& Carson, 2012; Pargament \& Mahoney, 2009). A pesar de algunos problemas metodológicos identificados (Schuurmans-Stekhoven, 2011), distintos estudios meta-analiticos reportan asociaciones significativas entre la religiosidad, la espiritualidad y la salud mental (Childs, 2010; Ellison \& Levin, 1998; Jim et al., 2015; Yonker, Schnabelrauch, \& Dehaan, 2012), tanto en relación a los trastornos del estado del ánimo (Payman \& Ryburn, 2010; Van Voorhees et al., 2008; Wachholtz \& Pargament, 2008), como a trastornos de ansiedad (Burdette, Ellison, Hill, 
$\&$ Glenn, 2009; Foley, Baillie, Huxter, Price, \& Sinclair, 2010; Korenromp, Page-Christiaens, van den Bout, Mulder, \& Visser, 2009) o al bienestar subjetivo (Lun \& Bond, 2013; Unterrainer, Ladenhauf, WallnerLiebmann, \& Fink, 2011) en diferentes poblaciones y contextos culturales (Fiorito \& Ryan, 2007; Lawler-Row \& Elliott, 2009; Toussaint, Williams, Musick, \& Everson, 2001).

\section{Salud Mental y espiritualidad.}

De acuerdo con King et al., (2013) las personas espirituales, en ausencia de un marco religioso, parecen experimentar una peor salud mental. Los autores realizaron un estudio en población general $(\mathrm{N}=7403)$ con el objeto de conocer las relaciones entre la espiritualidad, la religiosidad y los síntomas y diagnósticos psiquiátricos. En el estudio se informa que las personas que se consideran espirituales tienen una peor salud mental que las que los que no se consideran espirituales ni religiosas. Además, observaron que las personas religiosas resultan muy similares en cuanto a la prevalencia de trastornos mentales que las que no se consideran religiosas ni espirituales, excepto que resultan significativamente menos propensas a depender del consumo de drogas o alcohol.

De acuerdo con Coock y Powel (2013), el trabajo de King contradice la literatura en el área, que sugiere que la espiritualidad se asocia positivamente a la salud mental (Gall et al., 2005). Para Piedmont (2004), la relación entre la espiritualidad y la salud mental puede comprenderse en relación con la gestión del sentido de la mortalidad en el marco de la construcción de un sentido más amplio de significado personal en un contexto escatológico. Según el autor, sabiendo que va a morir, cada persona necesita construir un propósito y un significado para la vida. Las 
respuestas que cada persona encuentra a estas preguntas afectan sus elecciones personales, la trayectoria de sus historias de vida (Greenberg, Solomon, \& Pyszczynski, 1997), e idealmente, contribuyen a reunir los hilos dispares de la existencia de manera coherente y significativa, promoviendo una sensación de propósito (Piedmont, 2004). Para el autor, sin embargo, existen diferentes respuestas a estas preguntas existenciales, y algunas de las respuestas proporcionan un mayor bienestar que otras. Piedmont (2004) sugiere que una forma de clasificar las respuestas a estas preguntas existenciales remite al marco temporal que las personas emplean para otorgar significado a la vida. Por ejemplo, para el autor, algunas personas pueden percibirse en el contexto inmediato, en respuesta a las necesidades y demandas del presente (un horizonte de sucesos relativamente corto). Otros pueden comprender su vida como parte de una generación o cohorte específica, y el significado se desarrolla en relación a la forma en que estas personas perciben sus compromisos con otros miembros de su generación y de las generaciones que les siguen (un horizonte de eventos más moderado). Por último, otros pueden concebir sus vidas como parte de una vía ontológica eterna que implica responsabilidades para con los demás, tanto en el aquí y ahora como en otra vida (un horizonte de eventos de largo). Para Piedmont (2004), cuanto más amplio sea el horizonte de sucesos que se utiliza para crear un significado, mayor salud mental puede experimentar cada persona.

\section{Salud Mental y Religiosidad.}

Mientras que la mayoría de los estudios encuentran relaciones positivas entre la espiritualidad y la salud mental, la relación entre la religiosidad y la salud mental despierta mayores controversias (Koenig et al., 2012). Desde un enfoque cognitivo-conductual, James y Wells (2003) 
sugieren que esta disparidad en los resultados se debe a que la religiosidad puede ser comprendida como un modelo mental genérico que influye en la evaluación, la valoración y la lectura que se hace del mundo y de los eventos de la vida, pudiendo resultar tanto adaptativo como desadaptativo de acuerdo a su forma y contenido. Los autores identifican dos mecanismos que podrian explicar estas relaciones: (1) las creencias religiosas proveen modelos mentales genéricos que sirven de base para la evaluación de los eventos de la vida y (2) las creencias religiosas proveen una base para la autorregulación del proceso de pensamiento.

En relación al primer mecanismo, James y Wells (2003) sugieren que algunos modelos mentales podrian facilitar la comprensión de eventos estresantes de la vida. Por ejemplo, explicaciones religiosas en casos de lesiones fisicas por accidentes, como creer que una desgracia haya ocurrido con motivo de tener una lección que aprender, o para permitir que la víctima fuera puesta de ejemplo para otros, podrían promover una mayor salud mental (Maltby et al., 2010). Sin embargo, otros modelos mentales podrian contribuir a explicar la misma desgracia como un castigo de Dios por una falta de devoción, promoviendo un sentimiento de "abandono" por parte de Dios o de la comunidad religiosa (Pargament, Koenig, \& Perez, 2000). El segundo mecanismo supone que algunos comportamientos religiosos como el rezo o la meditación podrían contribuir, en algunos casos, a la autorregulación o la meta-cognición, mediante la reducción del foco en el sí mismo, la preocupación y el estrés, y por lo tanto, conducirian a percibir una mayor salud mental (James \& Wells, 2003). Sin embargo, no todas las formas de rezo se asociarian positivamente al bienestar. Por ejemplo, Poloma y Pendleton (1989) clasifican diferentes tipos de rezo entre los que las oraciones de petición, definidas como el pedido a Dios por objetos materiales, podian 
incrementar las rumiaciones y preocupaciones, asociándose a la percepción de un afecto negativo.

Por su parte, Koenig, King y Carson (2012) identifican algunos elementos de la religiosidad que podrian impactar negativamente en el BS, particularmente en aquellos sujetos que presentan altos niveles de neuroticismo. En primer lugar, la devoción excesiva por la práctica religiosa puede provocar conflictos interpersonales con individuos que no profesen la misma religión e incluso dentro de una pareja si ambos miembros no son igualmente religiosos. En el mismo sentido, ciertas interpretaciones de las escrituras religiosas pueden justificar el uso de la violencia contra otras personas o contra un miembro de la pareja cuando los contextos culturales o religiosos difieren (Ellison, Bartkowski, \& Anderson, 1999). Asimismo, involucrarse en ciertos cultos religiosos puede ocasionar dependencia emocional de un líder y aislamiento de la familia y el entorno íntimo. En segundo lugar, la religión puede promover un pensamiento rigidizado o dogmático y una dependencia excesiva de normas y reglas, restringiendo la autonomía individual y favoreciendo tendencias obsesivo compulsivas en las personas (Altemeyer \& Hunsberger, 1992). Por este motivo, si bien los fundamentalistas religiosos no necesariamente perciben sintomatología ansiosa o depresiva con mayor frecuencia que la población laica, la inflexibilidad cognitiva y el pensamiento dogmático que los caracteriza pueden promover numerosos conflictos interpersonales e intergrupales con otros miembros de la sociedad (Simkin \& Etchevers, 2014). Finalmente, ciertas creencias religiosas ortodoxas pueden entrar en conflicto con la necesidad de recurrir a tratamientos médicos, psiquiátricos o psicológicos aun cuando éstos son imprescindibles. En particular, la idea respecto de que la religión puede asociarse tanto de manera positiva como negativa a la 
satisfacción con la vida también ha sido evaluada en diferentes contextos culturales (Okulicz-Kozaryn, 2010).

\section{Religiosidad, espiritualidad y trastornos del estado de ánimo.}

La depresión puede considerarse un estado emocional que oscila entre una falta de motivación temporal hasta un trastorno mental de una alta mortalidad (Bostwick \& Pankratz, 2000). De manera similar a James y Wells (2003), Koenig, King y Carson (2012) sostienen que la religiosidad puede prevenir la depresión debido a que cierta lectura de las enseñanzas religiosas podría contribuir a afrontar diversos estresores de manera adaptativa. Sin embargo, los autores observan que las mismas enseñanzas también podrian promover altos estándares de conducta dificiles de sostener, lo que aparejaría sentimientos de culpa, fracaso y baja autoestima. A la vez, las dificultades para vivir de acuerdo a tales expectativas podrian conducir a experimentar rechazo social de la comunidad de pertenencia. Por este motivo, teóricamente, la religiosidad puede asociarse tanto a una mayor como a una menor depresión. En una revisión sistemática de la literatura, Koenig, King y Carson (2012) identifican 339 estudios que examinan la relación entre la religiosidad y la depresión publicados desde el año 2000. De estos trabajos 272 emplearon un diseño transversal, 45 prospectivo, y 22 experimental. Entre los estudios transversales identifican 170 (63\%) que reportan una asociación negativa entre la religiosidad y la depresión, 60 estudios (22\%) no encuentran ninguna asociación y 17 (6\%) una asociación positiva. Por otra parte, 22 estudios (8\%) reportaron resultados confusos debido a que la depresión se encontraba asociada de manera positiva a ciertos aspectos de la religiosidad y de manera negativa a otros. De los 45 estudios prospectivos, 21 trabajos (47\%) encontraron que una mayor religiosidad predice menores niveles de depresión, 5 estudios (11\%) 
encontraron que la religiosidad predice mayores niveles de depresión, 4 investigaciones $(9 \%)$ reportaron resultados confusos o contradictorios, y 14 trabajos (31\%) no encontraron ninguna relación entre las variables de estudio. Entre los 22 estudios experimentales, 14 trabajos (64\%) encontraron asociaciones positivas, 5 estudios no reportaron ninguna asociación y 2 una asociación negativa.

Entre los trabajos de mayor relevancia que encuentran una relación negativa, los autores destacan el trabajo de Strawbridge, Shema, Cohen y Kaplan (2001), quienes exploraron el vínculo de manera prospectiva en una muestra de 2.676 individuos de entre 17 y 65 años de edad entre 1965 y 1995. Los síntomas depresivos fueron evaluados a partir de una escala de 18 ítems similar a la CES-D y el inventario de depresión de Beck. De los participantes que se experimentaban sintomas depresivos en 1965 aquellos que atendian a servicios religiosos al menos dos o tres veces por semana experimentaron menores síntomas depresivos incluso al controlar edad, género, nivel educativo y autopercepciones de salud.

Entre los estudios que reportan asociaciones positivas se destaca el trabajo de Wijngaards-de Meij, Stroebe, Schut, Stroebe, van den Bout, van der Heijden, y Dijkstra (2005) quienes realizaron un estudio longitudinal explorando síntomas depresivos en una muestra de 219 parejas holandesas luego de la muerte de sus hijos. Los sujetos fueron entrevistados a los seis, trece y veinte meses de sus pérdidas. La religiosidad fue evaluada a partir de un solo item que relevaba afiliación religiosa. Los sintomas depresivos se evaluaron a partir de la subescala de 16 ítems del Hopkins Symptom Checklist-90. El análisis de regresión indicó que la afiliación religiosa de los padres predecía mayores síntomas depresivos.

Entre los estudios que identifican resultados mixtos más relevantes se encuentra el trabajo de Baetz, Griffin, Bowen, Koenig y Marcoux (2004), 
quienes emplearon un diseño transversal para explorar el vínculo entre diferentes características de la religiosidad y los sintomas depresivos en una muestra de 70.884 individuos desde los 16 años de edad en adelante. Para la evaluación de la religiosidad los autores emplearon diferentes ítems que relevaban frecuencia de asistencia a la iglesia, autopercepción de religiosidad y espiritualidad e importancia de los valores espirituales. Los síntomas depresivos fueron evaluados a partir del Composite International Diagnsostic interview -short form para Depresión mayor. De acuerdo con los resultados, la participación religiosa se asociaba negativamente a los sintomas depresivos, mientras que la centralidad de la religión para sus vidas se asociaba de manera positiva.

Por su parte, Shenesey (2009) evaluó la relación entre la espiritualidad, la religiosidad y los sintomas depresivos en una muestra compuesta por 143 estudiantes universitarios a partir de administrar la Escala de Evaluación de Espiritualidad y Sentimientos Religiosos (ASPIRES) y el Symptom Checklist-90-Revised (SCL-90-R). De acuerdo con los resultados, no se encontraron relaciones significativas entre la espiritualidad, la religiosidad y la salud mental.

\section{Religiosidad, Espiritualidad y trastornos de ansiedad.}

Koenig, King y Carson (2012) identifican 222 estudios que han explorado las relaciones entre religiosidad, espiritualidad y trastornos de ansiedad. De los 189 estudios transversales o prospectivos 82 trabajos (43\%) reportan asociaciones inversas, 22 artículos (12\%) reportan asociaciones positivas, 66 estudios (35\%) reportan ninguna asociación y 19 estudios $(10 \%)$ resultados confusos o complejos. De los 33 estudios excluidos de esta categoría, 8 estudios eran experimentales y 25 estudios clínicos. De los 8 estudios experimentales, 7 (88\%) reportaron una 
disminución de la ansiedad luego de intervenciones religiosas o espirituales mientras que de los 25 estudios clínicos 17 (64\%) reportó una disminución de la ansiedad luego de intervenciones religiosas o espirituales y 1 estudio (4\%) encontró un efecto negativo de intervenciones religiosas en los trastornos de ansiedad.

Entre los estudios que reportan una relación inversa se destaca el trabajo de Chen (2005), quien comparó los efectos de escribir acerca de eventos traumáticos desde una perspectiva religiosa y desde una perspectiva secular en la reducción de los sintomas del trastorno de estrés postraumático en 177 estudiantes de nivel superior, asignados a dos grupos. Se solicitó a los participantes que describieran experiencias traumáticas desde una perspectiva religiosa o secular de acuerdo a cada grupo en tres sesiones de escritura a lo largo de un mes. Para evaluar los sintomas de estrés postraumático se administró la Impact of Event Scale, mientras que la religiosidad fue relevada empleando la Organizational Religiousness Short Form y la Private Religious Practices Scale. De acuerdo al autor, inicialmente no se encontraron diferencias significativas entre ambos grupos en relación a la reducción de los síntomas de estrés postraumático. Sin embargo, mientras que la efectividad de la intervención secular disminuía cuanto mayor era la severidad del trauma, la intervención religiosa resultaba efectiva con independencia de la severidad del trauma. Por su parte, Braganza y Piedmont (2015) realizaron evaluaron la eficacia de un programa de intervención espiritual -Core Transformation Training- en 189 participantes de entre 18 y 65 años. Antes del entrenamiento y cinco semanas posteriores a la intervención los participantes respondieron una batería de instrumentos. Para la evaluación de la espiritualidad y la religiosidad se empleó la Escala de Evaluación de Espiritualidad y Sentimientos Religiosos, mientras que para evaluar trastornos del estado del ánimo y trastornos 
de ansiedad se empleó el Brief Symptom inventory - 18. De acuerdo con los autores, el programa de entrenamiento contribuyó a disminuir la experiencia de sintomas y a incrementar la espiritualidad.

En cuanto a los estudios que sugieren una relación positiva se destaca el trabajo de Miller, Forcehimes, O'Leary y LaNoue (2008) examinaron los efectos de la dirección espiritual en los síntomas de ansiedad en un estudio clínico en donde asignaron sesenta pacientes a un grupo en el que se administraron técnicas de orientación religiosa y un grupo secular. Después de cuatro meses los autores advirtieron que los sujetos del grupo asignado a intervenciones religiosas evidenciaban mayores síntomas de ansiedad, aunque a los doce meses no se observaran mayores diferencias intergrupales.

Entre quienes no encontraron diferencias significativas cabe mencionar el trabajo de Foley, Baillie, Huxter, Price y Sinclair, (2010) quienes examinaron los efectos un modelo de psicoterapia cognitiva basada en la meditación budista. Para la evaluación de la ansiedad, los investigadores administraron el Hamilton Anxiety Rating Scale luego del tratamiento y a los tres meses. De acuerdo a los autores, los resultados no fueron significativos al comparar el grupo experimental y el grupo control.

\section{Religiosidad, Espiritualidad y Bienestar Subjetivo.}

Koenig, King y Carson (2012) identifican 224 estudios publicados desde el año 2000 que relacionan religiosidad y bienestar subjetivo de los cuales el $68 \%$ refiere asociaciones positivas, 4\% resultados contradictorios, $27 \%$ ninguna asociación y dos estudios una relación negativa. 
Entre los trabajos de mayor relevancia, Koenig y Vaillant (2009) realizaron un estudio longitudinal durante 23 años en una muestra de ciudadanos norteamericanos $(\mathrm{N}=456)$ observando que la participación religiosa a los 47 años predecía el bienestar subjetivo a los 70 años. Por su parte, Wilkins, Piedmont y Magyar-Russell (2012) realizaron un estudio transversal en una muestra de 558 individuos que completaron la Escala de Evaluación de la Espiritualidad y los Sentimientos Religiosos (ASPIRES) -para la evaluación de la espiritualidad y de la religiosidad- y la Escala de Satisfacción con la Vida -para la evaluación del bienestar subjetivo-. De acuerdo con los resultados, el análisis de regresión contribuyó a comprobar que la espiritualidad -evaluada como un sexto factor del modelo de los cinco factores- promueve un mayor bienestar subjetivo. Por otra parte, Billig, Kohn y Levav (2006) realizaron un estudio correlacional en una muestra ciudadanos de la Franja de Gaza $(\mathrm{N}=267)$ concluyendo que aunque el afrontamiento religioso se encontraba asociado al distrés emocional, no se encontraba relacionado al bienestar subjetivo. A su vez, Wink y Scott (2005) examinaron la relación entre la religiosidad y bienestar subjetivo en una muestra de ciudadanos de California $(\mathrm{N}=155)$ reportando una asociación positiva entre ambos constructos.

\section{Problemas en la evaluación de la religiosidad y la espiritualidad.}

Los primeros trabajos en la conceptualización y la evaluación empírica de la religiosidad surgen a partir del interés por analizar el vínculo entre la religión y el prejuicio (Adorno, Frenkel-Brunswik, Levinson, \& Sanford, 1950). Aunque inicialmente la religiosidad es medida empleando simplemente indicadores tales como la frecuencia de asistencia a la iglesia o de la afiliación religiosa (Hill \& Pargament, 2003), posteriormente se desarrollan numerosos instrumentos de evaluación psicológica, como 
la Religious Orientation Scale (ROS) (Allport \& Ross, 1967), la Religious Coping Scale (RCOPE) (Pargament et al., 2000) o la Escala de Evaluación de Espiritualidad y Sentimientos Religiosos (ASPIRES) (Piedmont, 2004) que contribuyen a explorar los fenómenos religiosos con mayor rigurosidad, permitiendo identificar diferentes aspectos de las experiencias religiosas.

Sin embargo, de acuerdo con Hill (2012), si bien tales instrumentos han presentado propiedades psicométricas aceptables, las escalas aún mantienen considerables limitaciones. En primer lugar, la claridad conceptual en psicología de la religión para definir los constructos ha dificultado históricamente el desarrollo de escalas de evaluación psicológica (Belzen, 2012; Belzen \& Hood, 2006; Dittes, 1969). Estas dificultades pueden constatarse en la revisión de Koenig, King y Carson (2012), donde los diferentes estudios emplean distintos criterios para definir y evaluar estos términos, al punto que en algunos trabajos aún son utilizados como sinónimos. Tal como ha sido sugerido en la literatura (Zinnbauer et al., 1997), a diferencia de lo que ocurre con otros constructos psicológicos dentro de las corrientes principales de psicología -como la autoestima o la personalidad- para los que existe un mayor consenso en la literatura, las definiciones y operacionalizaciónes de la espiritualidad y de la religiosidad aún resultan confusas. El esfuerzo de Piedmont -quien fuera colaborador de Costa y McCrae durante la década del ochenta y noventa- por incluir la espiritualidad en el marco del modelo de los cinco factores de la personalidad supone un intento de abordar el término en el marco de otro constructo mejor consolidado en la disciplina. Sin embargo, si bien la propuesta ha despertado el interés de numerosos investigadores, en el campo de la psicología de la personalidad la espiritualidad aún no ha sido aceptada como un factor independiente del modelo (Saroglou, 2002, 2014a). 
En segundo lugar, la mayoría de las muestras empleadas para el armado de los instrumentos que suponen evaluar la espiritualidad y la religiosidad no son representativas (Hill \& Pargament, 2003). Por ejemplo, sobre una revisión de 24 escalas, Kapuscinski y Masters (2010) encuentran que solo una emplea una muestra representativa y que el $60 \%$ ha sido validada en población de estudiantes universitarios que no reflejan la diversidad de las experiencias religiosas del campo. Si bien en la revisión propuesta se identifican diferentes estudios que trabajan con población general o clínica, llama la atención la escasez de estudios realizados en población religiosa, y dentro de los estudios que sí la emplean se evidencia una mayor proporción de estudios en población católica o protestante. Si en psicología de la religión, tal como menciona Piedmont (2012) procura explorar aspectos generales, universales, de los fenómenos religiosos, una mayor representatividad de los distintas corrientes debería resultar ampliamente relevante, especialmente para el desarrollo de las técnicas de evaluación psicológicas que se diseñen para su relevamiento.

En tercer lugar, y de la misma manera, se ha criticado que en la construcción de escalas dificilmente se tienen en cuenta aspectos culturales de cada contexto (Chatters, Taylor, \& Lincoln, 2002; Y. Chen \& Chen, 2012; Nelson, 2012). En el mismo sentido, de acuerdo con Talak (2014), la concepción de la ciencia neutra propia de las corrientes principales en psicología rechaza en general el pluralismo de abordajes metodológicos, empleando categorias descriptivas que son en general cuantitativas. Así, se observa en la literatura que en el campo de la psicología de la religión la metodología cualitativa no es predominante ni siquiera al momento de la construcción de las técnicas de evaluación psicológicas. 
Por último, se ha señalado la escasez de programas de investigación específicos en psicología de la religión, por lo que la mayoría de los instrumentos de evaluación resultan particularmente breves, de modo de acotar la extensión de las baterías de instrumentos, limitándose a acompañar otras variables de mayor interés para cada estudio (Hill, 2012; Hill \& Pargament, 2003; King \& Crowther, 2004). Por este motivo, antes de la década de 1980, las escalas empleadas para evaluar la religiosidad y la espiritualidad dificilmente son utilizadas nuevamente tras su construcción inicial (Hill, 2012; Hill \& Hood, 1999). Tal como se informa en la literatura, en la revisión de Koenig, King y Carson (2012) un amplio porcentaje de estudios aún continúan evaluando la religiosidad y la espiritualidad a partir de unos pocos items, mientras que para la evaluación de los trastornos del estado de ánimo, de los trastornos de ansiedad, o del bienestar subjetivo, las técnicas psicométricas empleadas revestian mayor complejidad.

A modo de sintesis, resulta dificil de sistematizar los hallazgos reportados por los diferentes estudios debido a diferentes problemas relativos a la definición y operacionalización de los constructos numinosos. Posiblemente la disparidad en los resultados dependa en gran medida del modo en que cada investigador optara por evaluar la espiritualidad y la religiosidad.

\section{Conclusión}

La emergencia de la psicología de la religión a mediados de la década del ochenta se correspondió con el incremento de los estudios que se propusieran explorar el vínculo entre la espiritualidad, la religiosidad y la salud mental. Sin embargo, en la literatura especializada se han identificado numerosos inconvenientes en cuanto a la claridad 
conceptual de los términos espiritualidad y religiosidad $y$, consecuentemente, en el modo en que tales dificultades afectaron el desarrollo de técnicas de evaluación psicológicas. Tal como ha observado Talak (2014), las corrientes principales en psicologia se definen como apoliticas, neutras, objetivas y científicas, promoviendo un campo restringido en la forma de entender el bienestar humano y social, y de ahí sus limitaciones en la producción de conocimiento, a diferencia de otras ciencias sociales. Como sugiere la autora, si la perspectiva de estos enfoques es fundamentalmente individualista, y lo social y lo cultural se interpreta como algo externo al individuo, se terminan naturalizando muchos aspectos de la situación de los individuos considerándolos como rasgos individuales, que requieren ajustes adaptativos al medio. De esta forma, también se naturaliza el medio, ya que no se busca cambiarlo, solo se apunta a que el individuo cambie. Las corrientes principales de la psicología todavia no han adoptado la reflexividad -una exploración consciente de cómo nuestros valores y suposiciones afectan nuestras metas teóricas, nuestra metodología y actividades, y las interpretaciones que realizamos-, lo cual se fomenta en cambio en prácticas de investigación propias de ramas de las ciencias sociales (Talak, 2014).

Por lo expuesto, si bien se han identificado numerosos estudios que han examinado el vínculo entre la espiritualidad, la religiosidad y la salud mental posiblemente debido a esta falta de reflexividad propia de la disciplina, aún resulte necesario continuar trabajando en el modo en el que se definen y evalúan los términos, atendiendo una mayor complejidad de los enfoques metodológicos y epistemológicos implicados en el proceso de producción del conocimiento psicológico. 


\section{Referencias}

Adorno, T. W., Frenkel-Brunswik, E., Levinson, D. J., \& Sanford, R. N. (1950). The authoritarian personality. Oxford: Harpers.

Agilkaya, Z., \& Hood, R. W. (2012). Introduction to the Special Issue: Psychology of Religion in Turkey. Archive for the Psychology of Religion, 34(3), 281-284.

https://doi.org/10.1163/15736121-12341248

Ağılkaya-Şahin, Z., Streib, H., Ayten, A., \& Hood, R. W. (Eds.). (2015). Psychology of Religion in Turkey. Lieden: Brill. https://doi.org/10.1163/9789004290884

Allport, G. W. (1950). The individual and his religion. New York: Macmillan Company.

Allport, G. W., \& Ross, J. M. (1967). Personal religious orientation and prejudice. Journal of Personality and Social Psychology, 5(4), 432-443. https://doi.org/10.1037/h0021212

Altemeyer, B., \& Hunsberger, B. (1992). Authoritarianism, Religious Fundamentalism, Quest, and Prejudice. International Journal for the Psychology of Religion, 2(2), 113133. https: / / doi.org/10.1207/s15327582ijpr0202_5

American Psychiatric Association. (2013). Diagnostic and statistical manual of mental disorders (5th ed.). Washington: Author.

Anderson, J. R. (2015). The Social Psychology of Religion. Using scientific methodologies to understand religion. In Construction of Social Psychology: Advances in Psychology and Psychological Trends Series (pp. 173-185). Lisboa: Science Press.

Argyle, M., \& Beit-Hallahmi, Benjamin. (1975). The social psychology of religion. Oxford: Routledge \& Kegan Paul. Retrieved from http://psycnet.apa.org/psycinfo/1975-29429000

Aten, J. D., O’Grady, K. A., \& Worthington, E. L. (Eds.). (2011). The Psychology of Religion and Spirituality for Clinicians. New York: Routledge. https://doi.org/10.4324/9780203864920

Baetz, M., Griffin, R., Bowen, R., Koenig, H. G., \& Marcoux, E. (2004). The Association Between Spiritual and Religious Involvement and Depressive Symptoms in a Canadian Population. The Journal of Nervous and Mental Disease, 192(12), 818-822. https://doi.org/10.1097/01.nmd.0000146735.73827.85

Belzen, J. A. (2012). Psychology of religion. (J. A. Belzen, Ed.). New York: Springer. https://doi.org/10.1007/978-1-4614-1602-9

Belzen, J. A. (2015). Infrastructure in Early Psychology of Religion: The Fate of the First European Journals. International Psychology, Practice and Research, 5, 1-16.

Belzen, J. A., \& Hood, R. W. (2006). Methodological issues in the psychology of religion: toward another paradigm? The Journal of Psychology, 140(1), 5-28. https:/ / doi.org/10.3200/JRLP.140.1.5-28

Benson, P., \& Spilka, B. (1973). God Image as a Function of Self-Esteem and Locus of 
Control. Journal for the Scientific Study of Religion, 12(3), 297.

https://doi.org/10.2307/1384430

Bernt, F., Kasanzew, A., \& López, M. B. (2007). Una fe que haga justicia. orientaciones religiosas y sus correlatos sociales. Ciencias Psicológicas, 1(2), 171-178.

Billig, M., Kohn, R., \& Levav, I. (2006). Anticipatory stress in the population facing forced removal from the Gaza Strip. The Journal of Nervous and Mental Disease, 194(3), 195200. https://doi.org/10.1097/01.nmd.0000202489.78194.8d

Bostwick, J. M., \& Pankratz, V. S. (2000). Affective Disorders and Suicide Risk: A Reexamination. American Journal of Psychiatry, 157(12), 1925-1932. https://doi.org/10.1176/appi.ajp.157.12.1925

Braganza, D., \& Piedmont, R. L. (2015). The Impact of the Core Transformation Process on Spirituality, Symptom Experience, and Psychological Maturity in a Mixed Age Sample in India: A Pilot Study. Journal of Religion and Health, 54(3), 888-902. https://doi.org/10.1007/s10943-015-0049-y

Brown, I. T., Chen, T., Gehlert, N. C., \& Piedmont, R. L. (2013). Age and gender effects on the Assessment of Spirituality and Religious Sentiments (ASPIRES) scale: A crosssectional analysis. Psychology of Religion and Spirituality, 5(2), 90-98. https://doi.org/10.1037/a0030137

Burdette, A. M., Ellison, C. G., Hill, T. D., \& Glenn, N. D. (2009). "Hooking Up" at College: Does Religion Make a Difference? Journal for the Scientific Study of Religion, 48(3), 535551. https://doi.org/10.1111/j.1468-5906.2009.01464.x

Chalfant, H. P., \& Peek, C. W. (1983). Religious Affiliation, Religiosity and Racial Prejudice: A New Look at Old Relationships. Review of Religious Research, 25(2), 155. https:/ / doi.org/10.2307/3511492

Chatters, L. M., Taylor, R. J., \& Lincoln, K. D. (2002). Advances in the measurement of religiosity among older African Americans: implications for health and mental health researchers. In J. H. Skinner \& J. A. Teresi (Eds.), Multicultural measurement in older populations (pp. 199-220). New York: Springer.

Chen, Y., \& Chen, X. (2012). Methodological Issues in Psychology of Religion Research in the Chinese Context. Pastoral Psychology, 61(5), 671-683. https://doi.org/10.1007/s11089-012-0441-4

Chen, Y., Wang, J., Weng, H., \& Wang, X. (2012). History, Present Situation, and Problems of Chinese Psychology of Religion. Pastoral Psychology, 61(5-6), 641-654. https://doi.org/10.1007/s11089-011-0399-7

Chen, Y. Y. (2005). Written Emotional Expression and Religion: Effects on PTSD Symptoms. The International Journal of Psychiatry in Medicine, 35(3), 273-286. https:/ / doi.org/10.2190/2X0U-0CTB-Y877-5DRQ 
Childs, E. (2010). Religious Attendance and Happiness: Examining Gaps in the Current Literature-A Research Note. Journal for the Scientific Study of Religion, 49(3), 550-560. https://doi.org/10.1111/j.1468-5906.2010.01528.x

Ciarrocchi, J. W. (2012). Positive Psychology and Spirituality: A Virtue-Informed Approach to Well-Being. (L. J. Miller, Ed.), The Oxford Handbook of Psychology and Spirituality. Oxford University Press. https://doi.org/10.1093/oxfordhb/9780199729920.013.0027

Cochran, J. K., \& Beeghley, L. (1991). The Influence of Religion on Attitudes toward Nonmarital Sexuality: A Preliminary Assessment of Reference Group Theory. Journal for the Scientific Study of Religion, 30(1), 45. https://doi.org/10.2307/ 1387148

Cook, C. C. H., \& Powell, A. (2013). Spirituality is not bad for our mental health. The British Journal of Psychiatry, 202(5), 385-386. https://doi.org/10.1192/bjp.202.5.385

Costa, P. T., \& McCrae, R. R. (1980). Still stable after all these years: Personality as a key to some issues in adulthood and old age. In P. B. Baltes \& O. G. Brim (Eds.), Life span development and behavior (Vol. 3, pp. 65-102). New York: Academic Press.

De Souza, M., Francis, L. J., O’Higgins-Norman, J., \& Scott, D. (Eds.). (2009). International Handbook of Education for Spirituality, Care and Wellbeing (Vol. 3). Dordrecht: Springer Netherlands. https://doi.org/10.1007/978-1-4020-9018-9

Dein, S., Cook, C. C. H., Powell, A. E., \& Eagger, S. (2010). Research on religion, spirituality, and mental health: a review. Canadian Journal of Psychiatry Revue Canadienne de Psychiatrie, 54(5), 283-291. Retrieved from http://dx.doi.org/10.1192/pb.bp.109.025924

Diener, E. (2000). Subjective well-being: The science of happiness and a proposal for a national index. American Psychologist, 55(1), 34-43. https://doi.org/10.1037/0003066X.55.1.34

Diener, E. (2009). Positive Psychology: Past, Present, and Future. (C. R. Snyder \& S. J. Lopez, Eds.), Oxford handbook of positive psychology (2nd ed.). Oxford: Oxford University Press. https://doi.org/10.1093/oxfordhb/9780195187243.013.0002

Diener, E., Oishi, S., \& Lucas, R. E. (2009). Subjective well-being: The science of happiness and life satisfaction. In Lopez.Shane J \& C. R. Snyder (Eds.), Oxford handbook of positive psychology (pp. 187-194). Oxford.

Dittes, J. E. (1969). Psychology of religion. In G. Lindzey \& E. Aronson (Eds.), Handbook of social psychology (2nd ed., pp. 602-659).

Dueck, A., \& Han, B. (2014). Psychology of Religion in China. In D. A. Leeming (Ed.), Encyclopedia of Psychology and Religion (pp. 1429-1434). Boston, MA: Springer US. https://doi.org/10.1007/978-1-4614-6086-2_9351

Dy-Liacco, G. S., Piedmont, R. L., Murray-Swank, N. A., Rodgerson, T. E., \& Sherman, M. F. (2009). Spirituality and religiosity as cross-cultural aspects of human experience. 
Psychology of Religion and Spirituality, 1(1), 35-52. https://doi.org/10.1037/a0014937 Efklides, A., \& Moraitou, D. (Eds.). (2013). A Positive Psychology Perspective on Quality of Life (Vol. 51). Dordrecht: Springer Netherlands. https:/ / doi.org/10.1007/978-94-0074963-4

Ellis, A. (1986). Do Some Religious Beliefs Help Create Emotional Disturbance?

Psychotherapy in Private Practice, 4(4), 101-106.

https://doi.org/10.1300/J294v04n04_16

Ellison, C. G., Bartkowski, J. P., \& Anderson, K. L. (1999). Are There Religious Variations in Domestic Violence? Journal of Family Issues, 20(1), 87-113. https://doi.org/10.1177/019251399020001005

Ellison, C. G., \& Levin, J. S. (1998). The Religion-Health Connection: Evidence, Theory, and Future Directions. Health Education \& Behavior, 25(6), 700-720. https:/ / doi.org/10.1177/109019819802500603

Esperandio, M. R. G., \& Marques, L. F. (2014). The Psychology of Religion in Brazil. The International Journal for the Psychology of Religion, (July 2015), 1-17. https:/ / doi.org/ 10.1080/10508619.2014.952189

Fichter, J. H. (1962). Religion and Socialization among Children. Review of Religious Research, 4(1), 24. https://doi.org/10.2307/3509863

Fiorito, B., \& Ryan, K. (2007). Spirituality and Psychological Well-Being: A MediatorModerator Study. Review of Religious Research, 48(4), 341-368.

Foley, E., Baillie, A., Huxter, M., Price, M., \& Sinclair, E. (2010). Mindfulness-based cognitive therapy for individuals whose lives have been affected by cancer: A randomized controlled trial. Journal of Consulting and Clinical Psychology, 78(1), 72-79. https://doi.org/10.1037/a0017566

Fontaine, J. R. J., Duriez, B., Luyten, P., Corveleyn, J., \& Hutsebaut, D. (2005). Consequences of a Multidimensional Approach to Religion for the Relationship Between Religiosity and Value Priorities. International Journal for the Psychology of Religion, 15(2), 123-143. https://doi.org/10.1207/s15327582ijpr1502_2

Freud, S. (1979). Obras Completas. Buenos Aires: Amorrortu editores.

Gall, T. L., Charbonneau, C., Clarke, N. H., Grant, K., Joseph, A., \& Shouldice, L. (2005). Understanding the Nature and Role of Spirituality in Relation to Coping and Health: A Conceptual Framework. Canadian Psychology/Psychologie Canadienne, 46(2), 88-104. https:/ / doi.org/10.1037/h0087008

Galton, F. J. (1872). Statistical Inquiries into the Efficacy of Prayer. The Forthnightly Review, 12, 125-35. https://doi.org/10.1093/ije/dys 109

Gomes Esperandio, M. R., \& August, H. (2017). Quantitative Research in Psychology of Religion in Brazil. Revista Pistis Praxis, 9(1), 69. https://doi.org/10.7213/2175- 
1838.09.001.DS-TR03

Gorsuch, R. L. (1970). Rokeach's Approach to Value Systems and Social Compassion.

Review of Religious Research, 11(2), 139. https://doi.org/10.2307/3510277

Gorsuch, R. L. (1984). Measurement: The boon and bane of investigating religion. American

Psychologist, 39(3), 228-236. https://doi.org/10.1037/0003-066X.39.3.228

Greenberg, J., Solomon, S., \& Pyszczynski, T. (1997). Terror Management Theory of Self-

Esteem and Cultural Worldviews: Empirical Assessments and Conceptual Refinements.

In M. P. Zanna (Ed.), Advances in experimental social psychology (pp. 61-139). San

Diego: Academic Press. https://doi.org/10.1016/S0065-2601(08)60016-7

Greenfield, E. A., Vaillant, G. E., \& Marks, N. F. (2009). Do Formal Religious Participation

and Spiritual Perceptions Have Independent Linkages with Diverse Dimensions of Psychological Well-Being? Journal of Health and Social Behavior, 50(2), 196-212. https://doi.org/10.1016/j.biotechadv.2011.08.021.Secreted

Hill, P. C. (2012). Measurement Assessment and Issues in the Psychology of Religion and

Spirituality. In R. F. Paloutzian \& C. L. Park (Eds.), Handbook of Psychology of Religion and Spirituality (pp. 48-75). New York: The Guilford Press.

Hill, P. C., \& Hood, R. W. (1999). Measures of religiosity. Birmingham: Religious Education Press.

Hill, P. C., \& Pargament, K. I. (2003). Advances in the conceptualization and measurement of religion and spirituality: Implications for physical and mental health research. American Psychologist, 58(1), 64-74. https://doi.org/10.1037/0003-066X.58.1.64

Hill, P. C., Pargament, K. I., Hood, R. W., McCullough, Jr., M. E., Swyers, J. P., Larson, D. B., \& Zinnbauer, B. J. (2000). Conceptualizing Religion and Spirituality: Points of Commonality, Points of Departure. Journal for the Theory of Social Behaviour, 30(1), 5177. https://doi.org/10.1111/1468-5914.00119

Hökelekli, H. (2013). Religion, religiosity, and research in psychology of religion in Turkey: an assessment of locality and universality problem. Ilahiyat Studies: A Journal on Islamic and Religious Studies, 3(2), 223-245.

Hood Jr., R. W., \& Spilka, B. (2013). A Chronological Overview of the Psychology of Religion. Religious Studies and Theology, 31(2), 129-146.

https://doi.org/10.1558/rsth.v31i2.129

Huguelet, P., \& Koenig, H. G. (2009). Religion and Spirituality in Psychiatry. Cambridge: Cambridge University Press.

Idler, E. L., Boulifard, D. A., Labouvie, E., Chen, Y. Y., Krause, T. J., \& Contrada, R. J. (2009). Looking Inside the Black Box of "Attendance at Services": New Measures for Exploring an Old Dimension in Religion and Health Research. International Journal for the Psychology of Religion, 19(1), 1-20. https://doi.org/10.1080/10508610802471096 
James, A., \& Wells, A. (2003). Religion and mental health: towards a cognitive-behavioural framework. British Journal of Health Psychology, 8(3), 359-376.

https:// doi.org/10.1348/135910703322370905

James, W. (1890). The principles of psychology. New York: Holt.

James, W. (1902). The varieties of religious experience. New York: The Modern Library.

Jelen, T. G., \& Chandler, M. A. (1996). Patterns of Religious Socialization: Communalism, Associationalism and the Politics of Lifestyle. Review of Religious Research, 38(2), 142. https: / / doi.org/ 10.2307/3512338

Ji, C.-H. C., \& Ibrahim, Y. (2007). Islamic Doctrinal Orthodoxy and Religious Orientations: Scale Development and Validation. International Journal for the Psychology of Religion, 17(3), 189-208. https://doi.org/10.1080/10508610701402192

Jim, H. S. L., Pustejovsky, J. E., Park, C. L., Danhauer, S. C., Sherman, A. C., Fitchett, G., ... Salsman, J. M. (2015). Religion, spirituality, and physical health in cancer patients: A meta-analysis. Cancer, n/a-n/a. https://doi.org/10.1002/cncr.29353

Jung, C. G. (1938). Psychology and religion. Connecticut: Yale University Press.

Kapuscinski, A. N., \& Masters, K. S. (2010). The current status of measures of spirituality: A critical review of scale development. Psychology of Religion and Spirituality, 2(4), 191205. https://doi.org/10.1037/a0020498

King, J. E., \& Crowther, M. R. (2004). The measurement of religiosity and spirituality:

Examples and issues from psychology. Journal of Organizational Change Management, 17(1), 83-101. https://doi.org/10.1108/09534810410511314

King, M., Marston, L., McManus, S., Brugha, T., Meltzer, H., \& Bebbington, P. (2013). Religion, spirituality and mental health: results from a national study of English households. The British Journal of Psychiatry : The Journal of Mental Science, 202(1), 68-73. https://doi.org/10.1192/bjp.bp.112.112003

Kirkpatrick, L. A. (2013). Evolutionary Pscychology as a Foundation for the Psychology of Religion. In R. F. Paloutzian \& C. L. Park (Eds.), Handbook of the Psychology of Religion and Spirituality (pp. 118-138). New York: The Guilford Press.

Koenig, H. G. (2008). Concerns about measuring "spirituality" in research. The Journal of Nervous and Mental Disease, 196(5), 349-355.

https:/ / doi.org/10.1097/NMD.0b013e31816ff796

Koenig, H. G., King, D., \& Carson, V. B. (2012). Handbook of religion and health. London: Oxford University Press.

Koenig, L. B., \& Vaillant, G. E. (2009). A prospective study of church attendance and health over the lifespan. Health Psychology: Official Journal of the Division of Health Psychology, American Psychological Association, 28(1), 117-124. https://doi.org/10.1037/a0012984 
Korenromp, M. J., Page-Christiaens, G. C. M. L., van den Bout, J., Mulder, E. J. H., \& Visser, G. H. A. (2009). Adjustment to termination of pregnancy for fetal anomaly: a longitudinal study in women at 4, 8, and 16 months. American Journal of Obstetrics and Gynecology, 201(2), 160.e1-160.e7. https://doi.org/10.1016/j.ajog.2009.04.007

Krause, N., \& Ellison, C. G. (2007). Parental Religious Socialization Practices and SelfEsteem In Late Life. Review of Religious Research, 49(2), 109-127. Retrieved from http:/ / ezproxy.tamu.edu:2048/login?url=http://search.ebscohost.com/login.aspx?dir ect $=$ true $\& \mathrm{db}=\mathrm{rlh} \& \mathrm{AN}=28106688 \&$ site $=$ ehost-live

Krauss, S. E., Hamzah, A., Ismail, I. A., Suandi, T., Hamzah, S. R., Dahalan, D., \& Idris, F. (2012). Religious Socialization Among Malaysian Muslim Adolescents: A Family Structure Comparison. Review of Religious Research, 54(4), 499-518. https://doi.org/10.1007/s13644-012-0068-Z

Kwilecki, S. (1986). Religious Orientation and Personality: A Case Study. Review of Religious Research, 28(1), 16. https://doi.org/10.2307/3511334

LaBouff, J. P., Rowatt, W. C., Johnson, M. K., \& Finkle, C. (2012). Differences in Attitudes Toward Outgroups in Religious and Nonreligious Contexts in a Multinational Sample: A Situational Context Priming Study. International Journal for the Psychology of Religion, 22(1), 1-9. https://doi.org/10.1080/10508619.2012.634778

Lamers, S. (2012). Positive mental health: measurement, relevance and implications. https://doi.org/10.3990/1.9789036533706

Lamers, S., Westerhof, G. J., Glas, C. A. W., \& Bohlmeijer, E. T. (2015). The bidirectional relation between positive mental health and psychopathology in a longitudinal representative panel study. The Journal of Positive Psychology, 1-8. https://doi.org/10.1080/17439760.2015.1015156

Lawler-Row, K. A., \& Elliott, J. (2009). The Role of Religious Activity and Spirituality in the Health and Well-being of Older Adults. Journal of Health Psychology, 14(1), 43-52. https://doi.org/10.1177/1359105308097944

Leak, G. K., \& Randall, B. A. (1995). Clarification of the Link between Right-Wing Authoritarianism and Religiousness: The Role of Religious Maturity. Journal for the Scientific Study of Religion, 34(2), 245. https://doi.org/10.2307/1386769

Louden, S. H., \& Francis, L. J. (1999). The Personality Profile of Roman Catholic Parochial Secular Priests in England and Wales. Review of Religious Research, 41(1), 65. https:/ / doi.org/10.2307/3512427

Lu, L., \& Ke, J. (2012). A Concise History of Chinese Psychology of Religion. Pastoral Psychology, 61(5-6), 623-639. https://doi.org/10.1007/s11089-011-0395-y

Lun, V. M.-C., \& Bond, M. H. (2013). Examining the relation of religion and spirituality to subjective well-being across national cultures. Psychology of Religion and Spirituality, 
5(4), 304-315. https://doi.org/10.1037/a0033641

MacDonald, D. A. (2000). The Expressions of Spirituality Inventory: Test development, validation and scoring information.

MacDonald, D. A., Friedman, H. L., Brewczynski, J., Holland, D., Salagame, K. K. K., Mohan, K. K., ... Cheong, H. W. (2015). Spirituality as a Scientific Construct: Testing Its Universality across Cultures and Languages. Plos One, 10(3), 1-38. https://doi.org/10.1371/journal.pone.0117701

Maddux, J. E. (2008). Positive Psychology and the Illness Ideology: Toward a Positive Clinical Psychology. Applied Psychology, 57(s1), 54-70. https://doi.org/10.1111/j.1464-0597.2008.00354.x

Maltby, J., Lewis, C. A., Freeman, A., Day, L., Cruise, S. M., \& Breslin, M. J. (2010). Religion and health : the application of a cognitive-behavioural framework. Mental Health, Religion \& Culture, 13(7), 749-759. https://doi.org/10.1080/13674670802596930

McCrae, R. R., \& Costa, P. T. (1996). Toward a New Generation of Personality Theories: Theoretical Contexts for the Five-Factor Model. In J. S. Wiggins (Ed.), The five-factor model of personality: Theoretical perspectives. (pp. 51-87). New York: The Guilford Press.

McCrae, R. R., \& Costa, P. T. (2012). Personality in adulthood (2nd ed.). New York: The Guilford Press.

Melamed, A. R., Silverman, M. S., \& Lewis, G. J. (1974). Three Year Follow-up of Women Religious on the 16 Personality Factor Questionnaire. Review of Religious Research, 15(2), 64. https://doi.org/10.2307/3510235

Messay, B., Dixon, L. J., \& Rye, M. S. (2012). The relationship between Quest religious orientation, forgiveness, and mental health. Mental Health, Religion \& Culture, 15(3), 315-333. https://doi.org/10.1080/13674676.2011.574271

Miller, L. J. (2012). The Oxford handbook of psychology and spirituality. London: Oxford University Press.

Miller, W. R., Forcehimes, A., O’Leary, M. J., \& LaNoue, M. D. (2008). Spiritual direction in addiction treatment: Two clinical trials. Journal of Substance Abuse Treatment, 35(4), 434-442. https://doi.org/10.1016/j.jsat.2008.02.004

Miller, W. R., \& Thoresen, C. E. (1999). Spirituality and health. In W. R. Miller (Ed.), Integrating spirituality into treatment (pp. 3-18). Washington DC: American Psychological Association.

Muñoz, A. (2004). Cuestiones Epistemológicas Relativas al Estudio Psicológico de la Vivencia Religiosa. Psykhe (Santiago), 13(1). https://doi.org/10.4067/S071822282004000100011 
Musick, M. a, Traphagan, J. W. J., Koenig, H. G., \& Larson, D. B. (2000). Spirituality in physical health and aging. Journal of Adult Development, 7(2), 73-86. Retrieved from 10.1023/A:1009523722920\%5Cnhttp:// resources.library.brandeis.edu/login?url=http :/ / search.ebscohost.com/login.aspx?direct=true\&db=psyh\&AN=2000-12195$002 \&$ site $=$ ehost-live

Myers, D. G. (1993). The Pursuit of Happiness: Discovering the Pathway to Fulfillment, WellBeing, and Enduring Personal Joy. New York: Avon Books.

Myers, D. G. (2008). Religion and Human Flourishing. In M. Eid \& R. J. Larsen (Eds.), The Science of Subjective Well-Being (pp. 323-347). New York: The Guilford Press.

Nelson, J. M. (2012). A History of Psychology of Religion in the West: Implications for Theory and Method. Pastoral Psychology, 61(5-6), 685-710. https://doi.org/10.1007/s11089-011-0407-y

Okulicz-Kozaryn, A. (2010). Religiosity and life satisfaction across nations. Mental Health, Religion \& Culture, 13(2), 155-169. https://doi.org/10.1080/13674670903273801 Oman, D. (2013). Defining Religion and Spirituality. In R. F. Paloutzian \& C. L. Park (Eds.), Handbook of the Psychology of Religion and Spirituality (pp. 23-48). New York: The Guilford Press.

Paloutzian, R. (2017). Psychology of Religion in the World. Revista Pistis Praxis, 9(1), 15. https://doi.org/10.7213/2175-1838.09.001.DS01

Paloutzian, R. F., \& Park, C. L. (Eds.). (2013). Handbook of the Psychology of Religion and Spiritualty (2nd ed.). New York: The Guilford Press.

Pargament, K. I. (Ed.). (2013). APA Handbook of Psychology, Religion, and Spirituality. Washington DC: American Psychological Association.

Pargament, K. I., Koenig, H. G., \& Perez, L. M. (2000). The many methods of religious coping: Development and initial validation of the RCOPE. Journal of Clinical Psychology, 56(4), 519-543. https://doi.org/ 10.1002/(SICI) 1097-4679(200004)56:4<519::AIDJCLP6>3.0.CO;2-1

Pargament, K. I., \& Mahoney, A. (2009). Spirituality: The Search for the Sacred. (C. R. Snyder \& S. J. Lopez, Eds.) (2nd ed.). Oxford: Oxford University Press. https://doi.org/10.1093/oxfordhb/9780195187243.013.0058

Payman, V., \& Ryburn, B. (2010). Religiousness and recovery from inpatient geriatric depression: Findings from the PEJAMA Study. The Australian and New Zealand Journal of Psychiatry, 44(6), 560-567. https://doi.org/10.3109/00048671003606078

Piedmont, R. L. (1999). Does Spirituality Represent the Sixth Factor of Personality? Spiritual Transcendence and the Five-Factor Model. Journal of Personality, 67(6), 9851013. https://doi.org/10.1111/1467-6494.00080

Piedmont, R. L. (2004). Assessment of spirituality and religious sentiments, technical manual 
(1st ed.). Timonium, Maryland: Author.

Piedmont, R. L. (2009). The Contribution of Religiousness and Spirituality to Subjective Wellbeing and Satisfaction with Life. In M. Souza, L. J. Francis, J. O’Higgins-Norman, \& D. Scott (Eds.), International Handbook of Education for Spirituality, Care and Wellbeing (Vol. 3, pp. 89-105). Dordrecht: Springer Netherlands.

https:/ / doi.org/ 10.1007/978-1-4020-9018-9

Piedmont, R. L. (2012). Overview and Development of Measure of Numinous Constructs: The Assessment of Spirituality and Religious Sentiments (ASPIRES) Scale. In L. J. Miller (Ed.), The Oxford Handbook of Psychology and Spirituality (pp. 104-122). Oxford: Oxford University Press.

Piedmont, R. L. (2013). A short history of the Psychology of Religion and Spirituality: Providing growth and meaning for Division 36. Psychology of Religion and Spirituality, 5(1), 1-4. https://doi.org/10.1037/a0030878

Piedmont, R. L., Kennedy, M. C., Sherman, M. F., Sherman, N. C., \& Williams, J. E. G. (2008). A Psychometric Evaluation Of The Assessment Of Spirituality And Religious Sentiments (ASPIRES) Scale: Short Form. Research in the Social Scientific Study of Religion, 19, 163-182. https://doi.org/10.1163/ej.9789004166462.i-299.55

Poloma, M. M., \& Pendleton, B. F. (1989). Exploring Types of Prayer and Quality of Life: A Research Note. Review of Religious Research, 31(1), 46-53.

https://doi.org/10.2307/3511023

Rican, P., \& Janosova, P. (2010). Spirituality as a Basic Aspect of Personality: A CrossCultural Verification of Piedmont's Model. International Journal for the Psychology of Religion, 20(1), 2-13. https://doi.org/10.1080/10508610903418053

Ruckmick, C. A. (1920). The brevity book on psychology. Chicago: Brevity Publishers. Saroglou, V. (2002). Religion and the five factors of personality: A meta-analytic review. Personality and Individual Differences, 32(1), 15-25. https://doi.org/10.1016/S01918869(00)00233-6

Saroglou, V. (2011). Believing, Bonding, Behaving, and Belonging: The Big Four Religious Dimensions and Cultural Variation. Journal of Cross-Cultural Psychology, 42(8), 13201340. https://doi.org/10.1177/0022022111412267

Saroglou, V. (2014a). Introduction: Studying Religion in Personality and Social Psychology. In V. Saroglou (Ed.), Religion, personality, and social behavior (1st ed., pp. 1-28). New York: Taylor and Francis.

Saroglou, V. (Ed.). (2014b). Religion, Personality, and Social Behavior. New York: Taylor and Francis.

Sayés, J. A. (2006). Escatología. Madrid: Ediciones Palabra.

Schuurmans-Stekhoven, J. B. (2011). Is it God or Just the Data that Moves in Mysterious 
Ways? How Well-Being Research may be Mistaking Faith for Virtue. Social Indicators Research, 100(2), 313-330. https://doi.org/10.1007/s11205-010-9630-7

Seligman, M. (2002). Positive psychology, positive prevention, and positive therapy. In C. R.

Snyder \& S. J. Lopez (Eds.), Handbook of positive psychology (pp. 3-9). Oxford University Press.

Seligman, M., \& Csikszentmihalyi, M. (2000). Positive psychology. An introduction. The American Psychologist, 55(1), 5-14. https://doi.org/10.1177/0022167801411002

Seligman, M., \& Peterson, C. (2003). Positive clinical psychology. In L. G. Aspinwall \& U. M. Staudinger (Eds.), A psychology of human strengths: Fundamental questions and future directions for a positive psychology. (pp. 305-317). Washington DC: American Psychological Association.

Shenesey, J. W. (2009). An Examination of Spirituality, Religious Commitment, Personality and Mental Health. Tesis de Maestria, Universidad de South Alabama, Alabama.

Simkin, H. (2017). Adaptación y Validación al Español de la Escala de Evaluación de Espiritualidad y Sentimientos Religiosos (ASPIRES): la trascendencia espiritual en el modelo de los cinco factores. Universitas Psychologica, 16(2), 1-12. https://doi.org/10.11144/Javeriana.upsy16-2.aeee

Simkin, H., \& Etchevers, M. (2014). Religiosidad, espiritualidad y salud mental en el marco del Modelo de los Cinco Factores de la Personalidad. Acta Psiquiátrica Y Psicológica de América Latina, 60(4), 265-275.

Smith, C. B., Weigert, A. J., \& Thomas, D. L. (1979). Self-Esteem and Religiosity: An Analysis of Catholic Adolescents from Five Cultures. Journal for the Scientific Study of Religion, 18(1), 51. https://doi.org/10.2307/1385378

Spilka, B., \& Reynolds, J. F. (1965). Religion and Prejudice: A Factor-Analytic Study. Review of Religious Research, 6(3), 163. https://doi.org/10.2307/3509617

Strawbridge, W. J., Shema, S. J., Cohen, R. D., \& Kaplan, G. A. (2001). Religious attendance increases survival by improving and maintaining good health behaviors, mental health, and social relationships. Annals of Behavioral Medicine, 23(1), 68-74. https://doi.org/10.1207/S15324796ABM2301_10

Talak, A. M. (2014). Los valores en las explicaciones en psicología.

Toussaint, L. L., Williams, D. R., Musick, M. a., \& Everson, S. a. (2001). Forgiveness and health: Age differences in a US probability sample. Journal of Adult Development, 8(4), 249-257. https://doi.org/10.1023/A:1011394629736

Unterrainer, H.-F., Ladenhauf, K. H., Wallner-Liebmann, S. J., \& Fink, A. (2011). Different Types of Religious/Spiritual Well-Being in Relation to Personality and Subjective WellBeing. International Journal for the Psychology of Religion, 21(2), 115-126. https:/ / doi.org/10.1080/10508619.2011.557003 
Valle, E. (2017). A Brazilian Reading of Belzen's Towards Cultural Psychology of Religion. Pastoral Psychology, 66(3), 427-436. https://doi.org/10.1007/s11089-013-0587-8

Van Voorhees, B. W., Paunesku, D., Kuwabara, S. a., Basu, A., Gollan, J., Hankin, B. L., ... Reinecke, M. (2008). Protective and Vulnerability Factors Predicting New-Onset Depressive Episode in a Representative of U.S. Adolescents. Journal of Adolescent Health, 42(6), 605-616. https://doi.org/10.1016/j.jadohealth.2007.11.135

Wachholtz, A. B., \& Pargament, K. I. (2008). Migraines and meditation: does spirituality matter? Journal of Behavioral Medicine, 31(4), 351-366.

https://doi.org/10.1007/s10865-008-9159-2

Watson, P. J., Ghorbani, N., Davison, H. K., Bing, M. N., Hood, R. W., \& Ghramaleki, A. F. (2002). Negatively Reinforcing Personal Extrinsic Motivations: Religious Orientation, Inner Awareness, and Mental Health in Iran and the United States. International Journal for the Psychology of Religion, 12(4), 255-276.

https:/ /doi.org/10.1207/S15327582IJPR1204_04

Wesselmann, E. D., \& DeSouza, E. R. (2015). Bringing the Psychology of Religion and Spirituality Into Community Psychology. Journal of Prevention \& Intervention in the Community, 43(3), 163-164. https://doi.org/10.1080/10852352.2014.973274

Wijngaards-de Meij, L., Stroebe, M., Schut, H., Stroebe, W., van den Bout, J., van der Heijden, P., \& Dijkstra, I. (2005). Couples at Risk Following the Death of Their Child: Predictors of Grief Versus Depression. Journal of Consulting and Clinical Psychology, 73(4), 617-623. https://doi.org/10.1037/0022-006X.73.4.617

Wilkins, T. A., Piedmont, R. L., \& Magyar-Russell, G. M. (2012). Spirituality or religiousness: which serves as the better predictor of elements of mental health? Research in the Social Scientific Study of Religion, 23, 53-73.

Wink, P., \& Scott, J. (2005). Does religiousness buffer against the fear of death and dying in late adulthood? Findings from a longitudinal study. The Journals of Gerontology, 60(4), 207-214. https://doi.org/10.1093/geronb/60.4.P207

Woodworth, R. S. (1940). Psychology. New York: Henry Holt \& Co.

Yonker, J. E., Schnabelrauch, C. A., \& Dehaan, L. G. (2012). The relationship between spirituality and religiosity on psychological outcomes in adolescents and emerging adults: A meta-analytic review. Journal of Adolescence, 35(2), 299-314. https://doi.org/10.1016/j.adolescence.2011.08.010

Zinnbauer, B. J., \& Pargament, K. I. (2005). Religiousness and Spirituality. In R. F. Paloutzian \& C. L. Park (Eds.), Handbook of the Psychology of Religion and Spirituality (pp. 21-43). New York: The Guilford Press.

Zinnbauer, B. J., Pargament, K. I., Cole, B., Rye, M. S., Eric, M., Belavich, T. G., ... Butter, E. M. (1997). Religion and Spirituality: Unfuzzying the Fuzzy. Journal for the Scientific 
Study of Religion, 36(4), 549-564. https://doi.org/10.2307/1387689

Zinnbauer, B. J., Pargament, K. I., \& Scott, A. B. (1999). The Emerging Meanings of Religiousness and Spirituality: Problems and Prospects. Journal of Personality, 67(6), 889-919. https: / /doi.org/10.1111/1467-6494.00077 